\title{
Generalized gauge-invariant formulations of the strong-field approximation
}

\author{
Yulian V. Vanne and Alejandro Saenz \\ AG Moderne Optik, Institut für Physik, Humboldt-Universität zu Berlin, Hausvogteiplatz 5-7, D-10117 Berlin, Germany
}

(Dated: November 2, 2018)

\begin{abstract}
The gauge problem in the so-called strong-field approximation (SFA) describing atomic or molecular systems exposed to intense laser fields is investigated. Introducing a generalized gauge and partitioning of the Hamiltonian it is demonstrated that the $S$-matrix expansion obtained in the SFA depends on both gauge and partitioning in such a way that two gauges always yield the same $S$-matrix expansion, if the partitioning is properly chosen.
\end{abstract}

PACS numbers: $32.80 . \mathrm{Rm}, 33.80 . \mathrm{Rv}$

\section{INTRODUCTION}

Gauge-invariance is one of the fundamental concepts of electrodynamics. As a consequence it is, e.g., possible to formulate the interaction of charged particles with electromagnetic fields in different gauges. Although the choice of the gauge clearly influences parameters like the scalar or the vector potential, all physical quantities (observables) are independent of the gauge, if a complete treatment is performed. On the other hand, an approximate treatment often leads to gauge-dependent predictions for physical observables. One prominent example is given by the so-called strong-field approximation (SFA) for describing atomic or molecular systems exposed to intense laser fields that is also known as Keldysh-Faisal-Reiss (KFR) theory [1, 2, 3]. It is based on an (infinite) series expansion of the exact $S$ matrix describing the interaction of an atomic system with a laser pulse. Besides a phase factor, the $S$ matrix obtained from a calculation of the complete expansion should thus be independent on the chosen gauge, provided the series converge. Consequently, the corresponding observable transition probabilities (obtained from the squared absolute values of the $S$-matrix elements) should be gauge independent.

This gauge-independence of physical observables is usually lost, if only a truncated series is considered. This is the case for the SFA which is defined as the first term of the $S$-matrix expansion. In a number of investigations it has been shown that transition probabilities or rates predicted by either the length- or the velocity-gauge formulation of the SFA differ easily by one or two orders of magnitude for experimentally relevant laser parameters [4]. Furthermore, it was shown that the velocity-gauge SFA rate does not converge to the tunneling limit for weak fields, if longrange Coulomb interactions are present [5]. Recently, it was also demonstrated that there are pronounced qualitative differences between the energy distributions of the electrons ejected from, e. g., the $2 p$ state of hydrogen atoms exposed to intense laser fields, if they are calculated within the SFA and either the length or the velocity gauge [6]. The recent extensions of the SFA to molecular systems in velocity gauge [7], length gauge [8], or dressed length gauge [9] indicate similar or even more pronounced gauge dependencies for molecules. These findings have intensified the discussions whether the formulation of the SFA in one of the two gauges may be "superior" to the other. One approach to answer this question is rather pragmatic and based on a direct comparison of the SFA predictions in both gauges to either exact (numerical) solutions of the full time-dependent Schrödinger equation [6, 10] or to experimental results [11, 12]. Clearly, if there is no a priori reason that one of the two SFA formulations is superior to the other, the conclusions may vary depending on the considered atomic or molecular system (even its quantum state) as well as on laser-pulse parameters.

A second line of argumentation in favor of one of the two gauges is based on the question of "universality". For example, it has been argued that an evident limitation of the length-gauge formulation is the fact that in this case the predicted observables depend only on the scalar potential and thus a one-dimensional parameter, while the full description of an electromagnetic field requires in principle more than one dimension [13]. Very recently, Faisal proposes a "gauge-invariant" intense-field $S$-matrix theory that yields equal transition probabilities in length or velocity gauge; independent of the order of expansion [14]. Consequently, Faisal claims that his theory overcomes the above-mentioned long-standing discrepancy between the SFA in the two gauges. According to the findings in 14] the "gauge-invariant" and thus universal $S$-matrix theory appears to be equivalent to the traditional length-gauge formulation. Specifically, the first-order term reproduces exactly the Keldysh result [1] which was obtained in length gauge. In view of the popularity of the SFA for describing atomic and molecular ionization in intense laser fields (see, e. g., [15] or [16] and references therein), this is an important result.

Besides the evident appeal of a universal $S$-matrix formulation which would provide an end to the long-lasting debates on the choice of the appropriate gauge (for a very recent example, see [17, 18]), it appears, however, quite surprising that such a formulation should exist. In fact, Faisal derives in [19] an alternative $S$-matrix expansion that reproduces in length and velocity gauge the traditional velocity-gauge result. This alternative series expansion is 
obtained by a different partioning of the Hamiltonian. The particular results obtained in [14] and [19] immediately lead to the question of their generality. In the present work it is shown that it is possible to achieve an $S$-matrix expansion in agreement to any traditional SFA formulation in either length, velocity, or radiation gauge for an arbitrary choice of the gauge, if the Hamiltonian is correspondingly partitioned. In fact, introducing a generalized gauge transformation that includes the mentioned particular gauges as special cases, an in principle infinite set of different $S$-matrix expansions can be formulated. All of them can be shown to be achievable within any gauge as long as a proper partioning of the Hamiltonian is performed. Furthermore, the introduction of the generalized gauge allows to clearly demonstrate, how the choice of the gauge and the partitioning of the Hamiltonian describing the atomic or molecular system exposed to a laser field are connected with each other. This provides a much deeper insight in the gauge-problem of SFA that in fact turns out to be more properly described as an expansion problem.

In order to provide a clear definition of terms and notations, the following section gives a brief overview over the gauges relevant to the present work, including brief discussions on (local) gauge invariance, form invariance, and the dipole approximation. Most importantly, a generalized gauge is introduced in Sec.IIG, Sec.III discusses the wavefunction of a free-electron in an electromagnetic field and the influence of the chosen gauge on it. Equipped with these prerequisites, Sec.IV] discusses the $S$-matrix theory in different gauges. It represents thus the main results of this work in which it is demonstrated how various results ( $S$-matrix expansions) can be obtained using different combinations of gauge and partitioning.

\section{GAUGES}

In the validity regime of the SFA the laser intensities are so high that the photon density is also very high. Under these circumstances the number of photons can be treated as a continuous variable and the field can be described classically by using Maxwell's equations. Therefore, in the following a semi-classical theory will be used in which the radiation field is treated classically, but the atomic or molecular system is described using quantum mechanics. The influence of the quantum system on the external field is also neglected.

\section{A. Local gauge invariance}

A classical electromagnetic field is described by electric and magnetic field vectors, $\mathbf{F}(\mathbf{r}, t)$ and $\mathbf{B}(\mathbf{r}, t)$ or, alternatively, by the scalar and vector potentials $\Phi(\mathbf{r}, t)$ and $\mathbf{A}(\mathbf{r}, t)$. Consider a system consisting of an electron in a electrostatic potential $U(\mathbf{r})$ created by a nucleus (or some nuclei) which interacts with an external electromagnetic field. In semi-classical theory, the evolution of the system is governed by the time-dependent Schrödinger equation that satisfies local gauge invariance and is given in the coordinate representation with the minimal-coupling Hamiltonian by

$$
i \frac{\partial}{\partial t} \Psi_{\chi}(\mathbf{r}, t)=\left\{\frac{1}{2}\left[\mathbf{p}_{\mathrm{c}}+\mathbf{A}_{\chi}(\mathbf{r}, t)\right]^{2}-\Phi_{\chi}(\mathbf{r}, t)+U(\mathbf{r})\right\} \Psi_{\chi}(\mathbf{r}, t)
$$

where the subscript denotes the used gauge $\chi$ and the operator of canonical momentum is given independently of the gauge as $\mathbf{p}_{\mathrm{c}} \equiv-i \nabla$; a consequence of the definition of the minimal-coupling Hamiltonian. The word invariance means that if the wavefunction $\Psi_{\chi}$ and both potentials $\mathbf{A}_{\chi}$ and $\Phi_{\chi}$ are simultaneously transformed into a new gauge $\chi^{\prime}$ using the transformation recipes

$$
\begin{aligned}
\Psi_{\chi^{\prime}}(\mathbf{r}, t) & =\Psi_{\chi}(\mathbf{r}, t) e^{\left[i T_{\chi \rightarrow \chi^{\prime}}(\mathbf{r}, t)\right]} & \Psi_{\chi}(\mathbf{r}, t) & =\Psi_{\chi^{\prime}}(\mathbf{r}, t) e^{\left[i T_{\chi^{\prime} \rightarrow \chi}(\mathbf{r}, t)\right]} \\
\mathbf{A}_{\chi^{\prime}}(\mathbf{r}, t) & =\mathbf{A}_{\chi}(\mathbf{r}, t)-\nabla T_{\chi \rightarrow \chi^{\prime}}(\mathbf{r}, t) & \mathbf{A}_{\chi}(\mathbf{r}, t) & =\mathbf{A}_{\chi^{\prime}}(\mathbf{r}, t)-\nabla T_{\chi^{\prime} \rightarrow \chi}(\mathbf{r}, t) \\
\Phi_{\chi^{\prime}}(\mathbf{r}, t) & =\Phi_{\chi}(\mathbf{r}, t)+\frac{\partial}{\partial t} T_{\chi \rightarrow \chi^{\prime}}(\mathbf{r}, t) & \Phi_{\chi}(\mathbf{r}, t) & =\Phi_{\chi^{\prime}}(\mathbf{r}, t)+\frac{\partial}{\partial t} T_{\chi^{\prime} \rightarrow \chi}(\mathbf{r}, t)
\end{aligned}
$$

equation (11) is transformed into itself, but with $\Psi_{\chi} \rightarrow \Psi_{\chi^{\prime}}, \mathbf{A}_{\chi} \rightarrow \mathbf{A}_{\chi^{\prime}}$, and $\Phi_{\chi} \rightarrow \Phi_{\chi^{\prime}}$. The transformation functions $T_{\chi \rightarrow \chi^{\prime}}(\mathbf{r}, t)$ satisfy the relations

$$
T_{\chi \rightarrow \chi^{\prime}}=-T_{\chi^{\prime} \rightarrow \chi}, \quad T_{\chi \rightarrow \chi^{\prime}}+T_{\chi^{\prime} \rightarrow \chi^{\prime \prime}}=T_{\chi \rightarrow \chi^{\prime \prime}}
$$

Of course, physical quantities as the probability $P(\mathbf{r}, t)$ or the electric and magnetic fields $(\mathbf{F}(\mathbf{r}, t)$ and $\mathbf{B}(\mathbf{r}, t))$ are 
gauge independent, i. e.

$$
\begin{aligned}
& P(\mathbf{r}, t)=\left|\Psi_{\chi}(\mathbf{r}, t)\right|^{2}=\left|\Psi_{\chi^{\prime}}(\mathbf{r}, t)\right|^{2} \\
& \mathbf{F}(\mathbf{r}, t)=-\nabla \Phi_{\chi}(\mathbf{r}, t)-\frac{\partial}{\partial t} \mathbf{A}_{\chi}(\mathbf{r}, t)=-\nabla \Phi_{\chi^{\prime}}(\mathbf{r}, t)-\frac{\partial}{\partial t} \mathbf{A}_{\chi^{\prime}}(\mathbf{r}, t) \\
& \mathbf{B}(\mathbf{r}, t)=\nabla \times \mathbf{A}_{\chi}(\mathbf{r}, t)=\nabla \times \mathbf{A}_{\chi^{\prime}}(\mathbf{r}, t)
\end{aligned}
$$

\section{B. Form-invariant physical quantities}

A form-invariant quantity is defined as a quantity whose corresponding operator $G_{\chi}=G\left(\mathbf{A}_{\chi}, \Phi_{\chi}\right)$ is form invariant under a unitary transformation $\hat{\mathrm{T}}_{\chi \rightarrow \chi^{\prime}}=\exp \left[i T_{\chi \rightarrow \chi^{\prime}}(\mathbf{r}, t)\right]$, i. e.,

$$
G_{\chi^{\prime}} \equiv \hat{\mathrm{T}}_{\chi \rightarrow \chi^{\prime}} G_{\chi} \hat{\mathrm{T}}_{\chi \rightarrow \chi^{\prime}}^{\dagger}=G\left(\mathbf{A}_{\chi^{\prime}}, \Phi_{\chi^{\prime}}\right)
$$

The important difference between physical and non-physical quantities lies in the gauge invariance of the eigenvalues. The eigenvalues of a physical quantity (an observable) are identical in all gauges, whereas the eigenvalues of nonphysical quantities may depend on the chosen gauge. It can be shown that the eigenvalues of a form-invariant quantity are gauge-invariant, i. e. a physical quantity must be form-invariant. Consider the operator $G_{\chi}$ with the eigenvalues $g_{n}$ and the corresponding eigenstates $|\chi, n\rangle$,

$$
G_{\chi}|\chi, n\rangle=g_{n}|\chi, n\rangle
$$

The operator $G_{\chi^{\prime}}$ obtained by the unitary transformation (9) has the eigenstates $\left|\chi^{\prime}, n\right\rangle=\hat{\mathrm{T}}_{\chi \rightarrow \chi^{\prime}}|\chi, n\rangle$ and the same eigenvalues $g_{n}$

$$
G_{\chi^{\prime}}\left|\chi^{\prime}, n\right\rangle=\left(\hat{\mathrm{T}}_{\chi \rightarrow \chi^{\prime}} G_{\chi} \hat{\mathrm{T}}_{\chi \rightarrow \chi^{\prime}}^{\dagger}\right)\left(\hat{\mathrm{T}}_{\chi \rightarrow \chi^{\prime}}|\chi, n\rangle\right)=\hat{\mathrm{T}}_{\chi \rightarrow \chi^{\prime}} g_{n}|\chi, n\rangle=g_{n}\left|\chi^{\prime}, n\right\rangle
$$

Both $\mathbf{A}_{\chi}$ and $\Phi_{\chi}$ are not operators that correspond to physical quantities. The canonical momentum is also not form-invariant,

$$
\hat{\mathrm{T}}_{\chi \rightarrow \chi^{\prime}} \mathbf{p}_{\mathrm{c}}=\hat{\mathrm{T}}_{\chi \rightarrow \chi^{\prime}}^{\dagger} \mathbf{p}_{\mathrm{c}}-\nabla T_{\chi \rightarrow \chi^{\prime}}(\mathbf{r}, t)
$$

and, therefore, does not represent a physical measurable quantity, whereas the mechanical momentum

$$
\boldsymbol{\pi}_{\chi} \equiv \mathbf{p}_{\mathrm{c}}+\mathbf{A}_{\chi}(\mathbf{r}, t)
$$

is form-invariant,

$$
\hat{\mathrm{T}}_{\chi \rightarrow \chi^{\prime}} \boldsymbol{\pi}_{\chi} \hat{\mathrm{T}}_{\chi \rightarrow \chi^{\prime}}^{\dagger}=\mathbf{p}_{\mathrm{c}}-\nabla T_{\chi \rightarrow \chi^{\prime}}(\mathbf{r}, t)+\mathbf{A}_{\chi}=\mathbf{p}_{\mathrm{c}}+\mathbf{A}_{\chi^{\prime}}=\boldsymbol{\pi}_{\chi^{\prime}},
$$

and represents an observable. The electrostatic potential $U(\mathbf{r})$ also represents a physical quantity. The instantaneous energy operator of the system, $\mathcal{E}_{\chi}$,

$$
\mathcal{E}_{\chi} \equiv \frac{1}{2}\left[\mathbf{p}_{\mathrm{c}}+\mathbf{A}_{\chi}(\mathbf{r}, t)\right]^{2}+U(\mathbf{r})=\frac{\boldsymbol{\pi}_{\chi}^{2}}{2}+U(\mathbf{r})
$$

represents a physical quantity, since it is a function of only physical quantities: $\boldsymbol{\pi}_{\chi}$ and $U(\mathbf{r})$.

It is essential that both the total Hamiltonian of the system,

$$
\hat{\mathrm{H}}_{\chi} \equiv \frac{1}{2}\left[\mathbf{p}_{\mathrm{c}}+\mathbf{A}_{\chi}(\mathbf{r}, t)\right]^{2}-\Phi_{\chi}(\mathbf{r}, t)+U(\mathbf{r})=\mathcal{E}_{\chi}-\Phi_{\chi}(\mathbf{r}, t),
$$

and the field-free Hamiltonian,

$$
\hat{\mathrm{H}}_{0} \equiv \frac{\mathbf{p}_{\mathrm{c}}^{2}}{2}+U(\mathbf{r})
$$

are not form-invariant, although they determine the evolution of a physical system. 


\section{Radiation gauge}

The radiation gauge (labeled in the following by the subscript $\mathrm{R}$ ) is convenient, if no sources are presented. It is defined by the relations

$$
\nabla \cdot \mathbf{A}_{\mathrm{R}}=0, \quad \Phi_{\mathrm{R}}=0
$$

Therefore, one has $\nabla \cdot\left(\mathbf{A}_{\mathrm{R}} \Psi\right)=\mathbf{A}_{\mathrm{R}}(\nabla \Psi)+\left(\nabla \cdot \mathbf{A}_{\mathrm{R}}\right) \Psi=\mathbf{A}_{\mathrm{R}}(\nabla \Psi)$ and the total Hamiltonian of the system possesses the form

$$
\hat{\mathrm{H}}_{\mathrm{R}}=\mathbf{p}_{\mathrm{c}}^{2} / 2+\mathbf{A}_{\mathrm{R}} \cdot \mathbf{p}_{\mathrm{c}}+\mathbf{A}_{\mathrm{R}}^{2} / 2+U
$$

The vector potential in this gauge satisfies the wave equation and can be given by a superposition of monochromatic plane wave solutions. In the following, the vector function $\mathbf{A}(\mathbf{r}, t)$ specifies the vector potential $\mathbf{A}_{\mathrm{R}}(\mathbf{r}, t)$ in radiation gauge, so that

$$
\mathbf{F}(\mathbf{r}, t)=-\frac{\partial}{\partial t} \mathbf{A}(\mathbf{r}, t), \quad \mathbf{B}(\mathbf{r}, t)=\nabla \times \mathbf{A}(\mathbf{r}, t) .
$$

\section{Dipole approximation}

If the wavelength of the considered radiation is sufficiently long, the spatial variation of the radiation field across the system can be neglected. Assuming $\mathbf{A}(\mathbf{r}, t) \approx \mathbf{A}(t)$ one obtains

$$
\mathbf{F}=\mathbf{F}(t)=-\frac{\mathrm{d} \mathbf{A}(t)}{\mathrm{d} t}, \quad \mathbf{B}=0
$$

There exist two gauges, length and velocity gauge, which are extensively used in the context of the dipole approximation. It is, however, known that the conditions for a breakdown of the dipole approximation differ in different gauges. In order to explicitly highlight the terms which are neglected in the subsequent use of the dipole approximation, the general definitions of length and velocity gauges are given in the following subsections.

\section{E. Velocity gauge}

The velocity gauge (labeled in the following by the subscript V) is often used to remove the square of the vector potential from the total Hamiltonian. Note, the name velocity gauge is frequently used, if in fact the radiation gauge is meant. The reason is the equivalence of these two gauges in the weak-field limit in which the $A^{2}$ term can be neglected (with respect to the remaining terms). For strong fields this term is, however, not negligible and it is thus important to distinguish between these two gauges. The velocity gauge can in fact be obtained from the radiation gauge by means of the transformation function

$$
T_{\mathrm{R} \rightarrow \mathrm{V}}(\mathbf{r}, t)=\frac{1}{2} \int^{t} \mathbf{A}^{2}\left(\mathbf{r}, t^{\prime}\right) \mathrm{d} t^{\prime} \equiv \beta(\mathbf{r}, t)
$$

The vector and scalar potentials are given in the velocity gauge by

$$
\mathbf{A}_{\mathrm{V}}=\mathbf{A}-\nabla \beta, \quad \Phi_{\mathrm{V}}=\mathbf{A}^{2} / 2
$$

and the total Hamiltonian can be rewritten as

$$
\hat{\mathrm{H}}_{\mathrm{V}}=\mathbf{p}_{\mathrm{c}}^{2} / 2+\mathbf{A}_{\mathrm{V}} \cdot \mathbf{p}_{\mathrm{c}}+\mathrm{i} \Delta \beta+U
$$

where the identity $\nabla \cdot \mathbf{A}_{\mathrm{V}}=-\Delta \beta$ has been used.

Within the dipole approximation, the vector potential has the simpler form $\mathbf{A}_{\mathrm{V}}=\mathbf{A}(t)$ and Eq. (24) reduces to

$$
\hat{\mathrm{H}}_{\mathrm{V}}=\mathbf{p}_{\mathrm{c}}^{2} / 2+U+\mathbf{A}(t) \cdot \mathbf{p}_{\mathrm{c}}
$$




\section{F. Length gauge}

Another useful and popular gauge is the length gauge (labeled in the following by the subscript L). It is obtained from the radiation gauge, if the transformation function

$$
T_{\mathrm{R} \rightarrow \mathrm{L}}(\mathbf{r}, t)=\mathbf{A}(\mathbf{r}, t) \cdot \mathbf{r}
$$

is applied. This leads to the length-gauge vector and scalar potentials

$$
\mathbf{A}_{\mathrm{L}}=\mathbf{A}-\nabla(\mathbf{A} \cdot \mathbf{r})=-i \mathbf{L} \times \mathbf{A}, \quad \Phi_{\mathrm{L}}=-\mathbf{F} \cdot \mathbf{r}
$$

where $\mathbf{L} \equiv \mathbf{r} \times \mathbf{p}_{\mathrm{c}}$ is the canonical angular momentum. Within the dipole approximation, the vector potential in length gauge is simply zero. This leads to a simple form of the total Hamiltonian that is given in length gauge by

$$
\hat{\mathrm{H}}_{\mathrm{L}}=\mathbf{p}_{\mathrm{c}}^{2} / 2+U+\mathbf{F}(t) \cdot \mathbf{r}
$$

Note, that in this case the operators of the mechanical and canonical momentum coincide, $\boldsymbol{\pi}_{\mathrm{L}}=\mathbf{p}_{\mathrm{c}}$.

\section{G. Generalized gauge}

It can be shown that both the length and velocity gauges are only particular cases of a generalized gauge defined by an arbitrary set of parameters, $X=\left\{x_{1}, x_{2}\right\}$. This gauge, which will be referred to as $X$ gauge, is obtained via the transformation

$$
T_{\mathrm{R} \rightarrow X}(\mathbf{r}, t)=x_{1} \mathbf{A}(t) \cdot \mathbf{r}+x_{2} \beta(t)
$$

In the $X$ gauge the vector and scalar potentials are given as

$$
\mathbf{A}_{X}(t)=\left(1-x_{1}\right) \mathbf{A}(t), \quad \Phi_{X}=-x_{1} \mathbf{F}(t) \cdot \mathbf{r}+x_{2} \mathbf{A}^{2}(t) / 2
$$

This leads to the total Hamiltonian

$$
\hat{\mathrm{H}}_{X}=\mathbf{p}_{\mathrm{c}}^{2} / 2+U+\left(1-x_{1}\right) \mathbf{A}(t) \cdot \mathbf{p}_{\mathrm{c}}+x_{1} \mathbf{F}(t) \cdot \mathbf{r}+\left[\left(1-x_{1}\right)^{2}-x_{2}\right] \mathbf{A}^{2}(t) / 2 .
$$

In particular, $X=\{0,0\}, X=\{0,1\}$, and $X=\{1,0\}$ correspond to the total Hamiltonians in radiation gauge (19), velocity gauge (25), and length gauge (28), respectively.

\section{FREE ELECTRON IN A LASER FIELD}

\section{A. Volkov wave function}

Consider now a free electron in the presence of a laser field described by $\mathbf{A}(t)$. In velocity gauge the TDSE reads

$$
i \frac{\partial}{\partial t} \Psi_{\mathrm{V}}(\mathbf{r}, t)=\left[\mathbf{p}_{\mathrm{c}}^{2} / 2+\mathbf{A}(t) \cdot \mathbf{p}_{\mathrm{c}}\right] \Psi_{V}(\mathbf{r}, t) .
$$

The solution of Eq. (32) is the (non-relativistic) Volkov wavefunction

$$
\Psi_{\mathrm{V}, \mathbf{k}}(\mathbf{r}, t)=\exp \left[\mathrm{ik} \cdot\{\mathbf{r}-\boldsymbol{\alpha}(t)\}-\mathrm{i} E_{k} t\right], \quad E_{k}=k^{2} / 2
$$

where $\boldsymbol{\alpha}(t)=\int^{t} \mathbf{A}\left(t^{\prime}\right) \mathrm{d} t^{\prime}$ and $\delta$-function normalization is used. It is important to emphasize the physical meaning of the vector $\mathbf{k}$ and the scalar $E_{k}$ that are usually referred to as electron momentum and electron energy, respectively. In fact, $\mathbf{k}$ is the mean value of canonical momentum in velocity gauge, $\left\langle\mathbf{p}_{\mathrm{c}}\right\rangle_{\mathrm{V}} \equiv \mathbf{k}$. The mean value of mechanical momentum is on the other hand time-dependent and equal to $\mathbf{k}+\mathbf{A}(t)$. As was discussed above, this latter value is gauge-independent and will be denoted in the following by $\boldsymbol{\pi}$; thus one has $\boldsymbol{\pi}=\boldsymbol{\pi}(\mathbf{k}, t)=\mathbf{k}+\mathbf{A}(t)$. To stress the independence on a gauge, the vector function $\boldsymbol{\pi}$ has no subscript, in contrast to the operators $\boldsymbol{\pi}_{\mathrm{R}}, \boldsymbol{\pi}_{\mathrm{V}}$, or $\boldsymbol{\pi}_{\mathrm{L}}$. The mean value $\mathcal{E}(\mathbf{k}, t)=\boldsymbol{\pi}^{2}(\mathbf{k}, t) / 2$ of the instantaneous energy operator (15) is thus also time-dependent. 
Using the identity

$$
\frac{1}{2} \int^{t} \mathcal{E}\left(\mathbf{k}, t^{\prime}\right) \mathrm{d} t^{\prime}=E_{k} t+\mathbf{k} \cdot \boldsymbol{\alpha}(t)+\beta(t)
$$

the Volkov wavefunction can be written as either

$$
\Psi_{\mathrm{R}, \mathbf{k}}(\mathbf{r}, t)=\exp \left[\mathrm{ik} \cdot \mathbf{r}-\mathrm{i} \int^{t} \mathcal{E}\left(\mathbf{k}, t^{\prime}\right) \mathrm{d} t^{\prime}\right]
$$

or

$$
\Psi_{\mathrm{L}, \mathbf{k}}(\mathbf{r}, t)=\exp \left[\mathrm{i} \boldsymbol{\pi}(\mathbf{k}, t) \cdot \mathbf{r}-\mathrm{i} \int^{t} \mathcal{E}\left(\mathbf{k}, t^{\prime}\right) \mathrm{d} t^{\prime}\right]
$$

in radiation and length gauge, respectively.

In $X$ gauge one finds

$$
\Psi_{X, \mathbf{k}}(\mathbf{r}, t)=\exp \left[\mathrm{i} x_{1} \mathbf{A}(t) \cdot \mathbf{r}+\mathrm{i} x_{2} \beta(t)\right] \Psi_{\mathrm{R}, \mathbf{k}}(\mathbf{r}, t)=\mathrm{e}^{-\mathrm{i} \Theta_{X}(t)} \mathrm{e}^{\mathrm{i} \mathbf{k} \cdot \mathbf{r}}
$$

with

$$
\Theta_{X}(t)=E_{k} t+\mathbf{k} \cdot \boldsymbol{\alpha}(t)-x_{1} \mathbf{A}(t) \cdot \mathbf{r}-\left(x_{2}-1\right) \beta(t) .
$$

Note, in length gauge $\mathbf{k}$ can be seen purely as a parameter, since also the mean value of the canonical momentum $\left\langle\mathbf{p}_{\mathrm{c}}\right\rangle_{\mathrm{L}}$ is equal to $\boldsymbol{\pi}$. Nevertheless, the physical meaning of $\mathbf{k}$ becomes evident in the case of a linear-polarized monochromatic electromagnetic field.

\section{B. Linear-polarized monochromatic field}

For a vector potential given by $\mathbf{A}(t)=-\mathbf{A}_{0} \sin \omega t$ the corresponding electric field is $\mathbf{F}(t)=\mathbf{F}_{0} \cos \omega t$ with $\mathbf{F}_{0}=\omega \mathbf{A}_{0}$. In this case the mean value of the mechanical momentum is equal to $\boldsymbol{\pi}(\mathbf{k}, t)=\mathbf{k}-\mathbf{F}_{0} / \omega \sin \omega t$. Therefore, $\mathbf{k}$ is the value of the cycle-averaged $\boldsymbol{\pi}$. An electron with $\mathbf{k}=0$ quivers in the field around a single point in space.

The mean value $\mathcal{E}(\mathbf{k}, t)$ of the instantaneous energy operator (15) is time-dependent and given by

$$
\mathcal{E}(\mathbf{k}, t)=\frac{1}{2}\left(\mathbf{k}-\frac{\mathbf{F}_{0}}{\omega} \sin \omega t\right)^{2}=E_{k}-\mathbf{k} \cdot \frac{\mathbf{F}_{0}}{\omega} \sin \omega t-U_{p} \cos 2 \omega t+U_{p} .
$$

Therefore, its cycle-averaged value is equal to $E_{k}+U_{p}$. The ponderomotive energy $U_{p}=F_{0}^{2} /\left(4 \omega^{2}\right)$ is thus the cycle-averaged instantaneous energy of a quivering electron $(\mathbf{k}=0)$.

\section{FORMAL $S$-MATRIX FORMULATION OF SFA}

The following formulation of the $S$-matrix theory describing atomic and molecular systems in intense laser fields considers the case of a one-electron system for the sake of simplicity. The generalization to an arbitrary number of electrons is, however, straightforward. As a starting point the TDSE formulated in $X$ gauge is considered,

$$
\left(\mathrm{i} \frac{\partial}{\partial t}-\hat{\mathrm{H}}_{X}(t)\right)\left|\Psi_{X}(t)\right\rangle=0
$$

The electromagnetic field is absent before and after the pulse, i.e.

$$
\mathbf{A}(t)=\mathbf{F}(t)=0, \quad \hat{\mathrm{H}}_{X}(t)=\hat{\mathrm{H}}^{0} \quad \text { for } t<t_{i} \text { and } t>t_{f}
$$

Here, $\hat{\mathrm{H}}^{0}$ is the field-free Hamiltonian with eigenvalues $E_{\alpha}$ and eigenvectors $\left|\psi_{\alpha}\right\rangle$,

$$
\hat{\mathrm{H}}^{0}=\mathbf{p}_{\mathrm{c}}^{2} / 2+U, \quad \hat{\mathrm{H}}^{0}\left|\psi_{\alpha}\right\rangle=E_{\alpha}\left|\psi_{\alpha}\right\rangle .
$$

(The index $\alpha$ denotes discrete as well as continuum states and is thus itself either discrete or continuous.) 
To describe the action of the pulse on the system, complete and orthonormal initial- and final-state basis sets are introduced. The initial-state basis set is given by $\left|\psi_{\alpha}\left(t_{i}\right)\right\rangle=\mathrm{e}^{-\mathrm{i} E_{\alpha} t_{i}}\left|\psi_{\alpha}\right\rangle$ where the phase factor is introduced for convenience. The final-state basis set is given by plane waves with momentum $\mathbf{k}$, again for convenience multiplied by a phase factor, and depends both on the adopted gauge and on $\mathbf{k}$,

$$
\left|\Psi_{X, \mathbf{k}}\left(t_{f}\right)\right\rangle=\mathrm{e}^{-\mathrm{i} \Theta_{X}\left(t_{f}\right)}|\mathbf{k}\rangle
$$

The phase (see Eqs. (38) and (41))

$$
\Theta_{X}\left(t_{f}\right)=E_{k} t_{f}+\mathbf{k} \cdot \boldsymbol{\alpha}\left(t_{f}\right)-\left(x_{2}-1\right) \beta\left(t_{f}\right)
$$

is $\mathbf{r}$ independent but depends on the used gauge, $\mathbf{k}$, and the pulse. Note, that the for reasons of convenience introduced phase factors add only constant phases in the transition amplitudes and do not alter transition probabilities.

The probability amplitude of a transition from an initial state $\left|\psi_{\alpha}\left(t_{i}\right)\right\rangle$ to a final state $\left|\Psi_{X, \mathbf{k}}\left(t_{f}\right)\right\rangle$ is given by

$$
S_{\mathbf{k} \alpha}=\mathrm{i}\left\langle\Psi_{X, \mathbf{k}}\left(t_{f}\right)\left|\hat{\mathrm{G}}_{X}\left(t_{f}, t_{i}\right)\right| \psi_{\alpha}\left(t_{i}\right)\right\rangle
$$

where the propagator $\hat{\mathrm{G}}_{X}\left(t, t^{\prime}\right)$ is associated with $\hat{\mathrm{H}}_{X}(t)$ by the inhomogeneous equation

$$
\left(\mathrm{i} \frac{\partial}{\partial t}-\hat{\mathrm{H}}_{X}(t)\right) \hat{\mathrm{G}}_{X}\left(t, t^{\prime}\right)=\delta\left(t-t^{\prime}\right) .
$$

To obtain a systematic expansion of the transition amplitudes of interest it is convenient to express the total propagator $\hat{\mathrm{G}}_{X}$ of the system in terms of a partial propagator, defined by a partitioning of the total Hamiltonian. The choice of the partitioning is made in such a way that the partial propagator can be expressed analytically, i. e. the Schrödinger equation with the corresponding partial Hamiltonian is solvable.

A first class of Hamiltonians that leads to analytical solutions is the one describing a free electron in the field. As was discussed in Sec. III, such Hamiltonians are gauge-dependent and their solutions are given by Volkov states. The partitioning of $\hat{\mathrm{H}}_{X}$ using the free-electron Hamiltonian in $X$ gauge, $\hat{\mathrm{H}}_{X}^{\mathrm{f}}$, is given by

$$
\hat{\mathrm{H}}_{X}=\hat{\mathrm{H}}_{X}^{\mathrm{f}}+U
$$

The corresponding propagator can be written analytically using the solutions $\left|\Psi_{X, \mathbf{k}}(t)\right\rangle$,

$$
\hat{\mathrm{G}}_{X}^{\mathrm{f}}\left(t, t^{\prime}\right)=-i \theta\left(t-t^{\prime}\right) \sum_{\mathbf{k}}\left|\Psi_{X, \mathbf{k}}(t)\right\rangle\left\langle\Psi_{X, \mathbf{k}}\left(t^{\prime}\right)\right|,
$$

where $\theta(x)$ is the step function. From Eq. (48) follows

$$
-i\left\langle\Psi_{X, \mathbf{k}}\left(t_{f}\right)\right| \hat{\mathrm{G}}_{X}^{\mathrm{f}}\left(t_{f}, t\right)=\left\langle\Psi_{X, \mathbf{k}}(t)\right| \text { for } t<t_{f} .
$$

Another Hamiltonian that can be used for the partitioning is the field-free Hamiltonian $\hat{\mathrm{H}}^{0}$. It is, however, only a special case of the class of Hamiltonians, which will be referred to as generalized field-free Hamiltonians and will be considered in the following subsection.

\section{A. Generalized field-free Hamiltonian}

The generalized field-free Hamiltonian

$$
\hat{\mathrm{H}}_{\gamma}^{0}=\mathrm{e}^{\mathrm{i} \gamma(\mathbf{r}, t)} \hat{\mathrm{H}}^{0} \mathrm{e}^{-\mathrm{i} \gamma(\mathbf{r}, t)}-\frac{\partial \gamma(\mathbf{r}, t)}{\partial t}=\hat{\mathrm{H}}^{0}+\frac{\mathrm{i}}{2} \Delta \gamma-(\nabla \gamma) \cdot \mathbf{p}_{\mathrm{c}}+\frac{1}{2}(\nabla \gamma)^{2}-\frac{\partial \gamma}{\partial t}
$$

is defined with the aid of an arbitrary function $\gamma(\mathbf{r}, t)$ in such a way that it reduces for $\gamma=0$ to the field-free Hamiltonian $\hat{H}^{0}$. Solutions of the TDSE with $\hat{H}_{\gamma}^{0}$ can be expanded in terms of the solutions of the TDSE with $\hat{H}^{0}$ as

$$
\left|\Psi_{\gamma, \alpha}(t)\right\rangle=\mathrm{e}^{\mathrm{i} \gamma(\mathbf{r}, t)} \mathrm{e}^{-\mathrm{i} E_{\alpha} t}\left|\psi_{\alpha}\right\rangle .
$$

In general, the function $\gamma(\mathbf{r}, t)$ can be chosen independent of the gauge that is used to formulate the TDSE. Consider a particular choice of $\gamma(\mathbf{r}, t)$ parameterized by a set of parameters, $\lambda=\left\{\lambda_{1}, \lambda_{2}\right\}$,

$$
\gamma_{\lambda}(\mathbf{r}, t)=\lambda_{1} \mathbf{A}(t) \cdot \mathbf{r}+\lambda_{2} \beta(t)
$$


The corresponding generalized field-free Hamiltonian (the subscript $\lambda$ is adopted instead of $\gamma_{\lambda}$ for the sake of notational simplicity) is then given by

$$
\hat{\mathrm{H}}_{\lambda}^{0}=\hat{\mathrm{H}}^{0}-\lambda_{1} \mathbf{A}(t) \cdot \mathbf{p}_{\mathrm{c}}+\lambda_{1} \mathbf{F}(t) \cdot \mathbf{r}+\left(\lambda_{1}^{2}-\lambda_{2}\right) \mathbf{A}^{2}(t) / 2 .
$$

Note, for all choices of $\lambda$ the Hamiltonian $\hat{H}_{\lambda}^{0}$ gives an equivalent description of the evolution before and after the pulse, since for those times both $\mathbf{A}(t)$ and $\mathbf{F}(t)$ are equal to zero. Different choices of $\lambda$ yield, however, different partial propagators $\hat{H}_{\lambda}^{0}$ during the pulse that can be written analytically as

$$
\hat{\mathrm{G}}_{\lambda}^{0}\left(t, t^{\prime}\right)=-i \theta\left(t-t^{\prime}\right) \sum_{\alpha}\left|\Psi_{\lambda, \alpha}(t)\right\rangle\left\langle\Psi_{\lambda, \alpha}\left(t^{\prime}\right)\right| .
$$

In order to express the total propagator $\hat{\mathrm{G}}_{X}$ in terms of $\hat{\mathrm{G}}_{\lambda}^{0}$, the total Hamiltonian is partitioned in two parts,

$$
\hat{\mathrm{H}}_{X}=\hat{\mathrm{H}}_{\lambda}^{0}+V_{X, \lambda}^{0}
$$

where the interaction operator $V_{X, \lambda}^{0}$ is given by

$$
\begin{gathered}
V_{X, \lambda}^{0}(t)=\left(1-x_{1}+\lambda_{1}\right) \mathbf{A}(t) \cdot \mathbf{p}_{\mathrm{c}}+\left(x_{1}-\lambda_{1}\right) \mathbf{F}(t) \cdot \mathbf{r} \\
+\left[\left(1-x_{1}\right)^{2}-x_{2}-\lambda_{1}^{2}+\lambda_{2}\right] \mathbf{A}^{2}(t) / 2
\end{gathered}
$$

It is worth reminding that both sets of parameters, $X=\left\{x_{1}, x_{2}\right\}$ and $\lambda=\left\{\lambda_{1}, \lambda_{2}\right\}$, are independent of each other. Therefore, the same interaction operator can be obtained for different $X$ gauges, if the $\lambda$ parameters are appropriately chosen. It can be shown, for example, that

$$
\begin{aligned}
& V_{\mathrm{R},\{-1,0\}}^{0}=V_{\mathrm{V},\{-1,1\}}^{0}=V_{\mathrm{L},\{0,0\}}^{0}=\mathbf{F}(t) \cdot \mathbf{r} \\
& V_{\mathrm{R},\{0,-1\}}^{0}=V_{\mathrm{V},\{0,0\}}^{0}=V_{\mathrm{L},\{1,1\}}^{0}=\mathbf{A}(t) \cdot \mathbf{p}_{\mathrm{c}} \\
& V_{\mathrm{R},\{0,0\}}^{0}=V_{\mathrm{V},\{0,1\}}^{0}=V_{\mathrm{L},\{1,2\}}^{0}=\mathbf{A}(t) \cdot \mathbf{p}_{\mathrm{c}}+\mathbf{A}^{2}(t) / 2
\end{aligned}
$$

Since $\left|\Psi_{\lambda, \alpha}\left(t_{i}\right)\right\rangle=\left|\psi_{\alpha}\left(t_{i}\right)\right\rangle$, Eq. (154) yields

$$
i \hat{\mathrm{G}}_{\lambda}^{0}\left(t, t_{i}\right)\left|\psi_{\alpha}\left(t_{i}\right)\right\rangle=\left|\Psi_{\lambda, \alpha}(t)\right\rangle, \quad \text { for } t>t_{i}
$$

\section{B. Matrix elements}

It will now be shown that most of the matrix elements of interest depend at most on the two parameters $v=\left\{v_{1}, v_{2}\right\}$ with $v_{1}=1+\lambda_{1}-x_{1}$ and $v_{2}=\lambda_{2}-x_{2}$.

Indeed, one finds for different matrix elements the relations

$$
\begin{gathered}
\left\langle\Psi_{X, \mathbf{k}}(t) \mid \Psi_{\lambda, \alpha}(t)\right\rangle=\left\langle\mathbf{k}\left|\mathrm{e}^{\mathrm{i} \Omega_{v \mathbf{k} \alpha}(t)}\right| \psi_{\alpha}\right\rangle \\
\left\langle\Psi_{X, \mathbf{k}}(t)|U| \Psi_{\lambda, \alpha}(t)\right\rangle=\left\langle\mathbf{k}\left|\mathrm{e}^{\mathrm{i} \Omega_{v \mathbf{k} \alpha}(t)} U\right| \psi_{\alpha}\right\rangle \\
\left\langle\Psi_{\lambda, \alpha^{\prime}}(t)\left|V_{X, \lambda}^{0}(t)\right| \Psi_{\lambda, \alpha}(t)\right\rangle=\mathrm{e}^{\mathrm{i}\left(E_{\alpha^{\prime}}-E_{\alpha}\right) t}\left\langle\psi_{\alpha^{\prime}}\left|\bar{V}_{v}^{0}(t)\right| \psi_{\alpha}\right\rangle,
\end{gathered}
$$

and

$$
\left\langle\Psi_{X, \mathbf{k}}(t)\left|V_{X, \lambda}^{0}(t)\right| \Psi_{\lambda, \alpha}(t)\right\rangle=\left\langle\mathbf{k}\left|\mathrm{e}^{\mathrm{i} \Omega_{v \mathbf{k} \alpha}(t)} \bar{V}_{v}^{0}(t)\right| \psi_{\alpha}\right\rangle
$$

where

$$
\begin{aligned}
\bar{V}_{v}^{0}(t) & =V_{X, \lambda}^{0}(t)+\lambda_{1}\left(1-x_{1}+\lambda_{1}\right) \mathbf{A}^{2}(t) \\
& =v_{1} \mathbf{A}(t) \cdot \mathbf{p}_{\mathrm{c}}+\left(1-v_{1}\right) \mathbf{F}(t) \cdot \mathbf{r}+\left(v_{1}^{2}+v_{2}\right) \mathbf{A}^{2}(t) / 2
\end{aligned}
$$

and

$$
\begin{aligned}
\Omega_{v \mathbf{k} \alpha}(t) & =\Theta_{X}(t)+\gamma_{\lambda}(\mathbf{r}, t)-E_{\alpha} t \\
& =\left(E_{k}-E_{\alpha}\right) t+\mathbf{k} \cdot \boldsymbol{\alpha}(t)+\left(v_{1}-1\right) \mathbf{A}(t) \cdot \mathbf{r}+\left(v_{2}+1\right) \beta(t)
\end{aligned}
$$


Finally, the matrix element

$$
\left\langle\Psi_{X, \mathbf{k}^{\prime}}(t)|U| \Psi_{X, \mathbf{k}}(t)\right\rangle=\mathrm{e}^{\mathrm{i}\left(E_{k^{\prime}}-E_{k}\right) t+\mathrm{i}\left(\mathbf{k}^{\prime}-\mathbf{k}\right) \cdot \boldsymbol{\alpha}(t)}\left\langle\mathbf{k}^{\prime}|U| \mathbf{k}\right\rangle
$$

is independent both on gauge and partitioning.

As a consequence of these properties of the matrix elements the transition amplitude depends only on $v$, as is shown below.

\section{S-matrix series}

The operator $\hat{\mathrm{G}}_{X}\left(t, t^{\prime}\right)$ can be expanded either in terms of the operator $\hat{\mathrm{G}}_{\lambda}^{0}\left(t, t^{\prime}\right)$,

$$
\hat{\mathrm{G}}_{X}\left(t, t^{\prime}\right)=\hat{\mathrm{G}}_{\lambda}^{0}\left(t, t^{\prime}\right)+\int \mathrm{d} t_{1} \hat{\mathrm{G}}_{X}\left(t, t_{1}\right) V_{X, \lambda}^{0}\left(t_{1}\right) \hat{\mathrm{G}}_{\lambda}^{0}\left(t_{1}, t^{\prime}\right),
$$

or in terms of the operator $\hat{\mathrm{G}}_{X}^{\mathrm{f}}\left(t, t^{\prime}\right)$,

$$
\hat{\mathrm{G}}_{X}\left(t, t^{\prime}\right)=\hat{\mathrm{G}}_{X}^{\mathrm{f}}\left(t, t^{\prime}\right)+\int \mathrm{d} t_{1} \hat{\mathrm{G}}_{X}^{\mathrm{f}}\left(t, t_{1}\right) U \hat{\mathrm{G}}_{X}\left(t_{1}, t^{\prime}\right) .
$$

Substitution of Eq. (69) in (68) yields

$$
\begin{aligned}
\hat{\mathrm{G}}_{X}\left(t, t^{\prime}\right) & =\hat{\mathrm{G}}_{\lambda}^{0}\left(t, t^{\prime}\right)+\int \mathrm{d} t_{1} \hat{\mathrm{G}}_{X}^{\mathrm{f}}\left(t, t_{1}\right) V_{X, \lambda}^{0}\left(t_{1}\right) \hat{\mathrm{G}}_{\lambda}^{0}\left(t_{1}, t^{\prime}\right) \\
& +\iint \mathrm{d} t_{2} \mathrm{~d} t_{1} \hat{\mathrm{G}}_{X}^{\mathrm{f}}\left(t, t_{2}\right) U \hat{\mathrm{G}}_{X}\left(t_{2}, t_{1}\right) V_{X, \lambda}^{0}\left(t_{1}\right) \hat{\mathrm{G}}_{\lambda}^{0}\left(t_{1}, t^{\prime}\right) .
\end{aligned}
$$

A further substitution of either (68) or (69) in Eq. (70) results in a series expansion of $\hat{\mathrm{G}}_{X}\left(t, t^{\prime}\right)$. Inserting this expansion in Eq. (45) generates the $S$-matrix series for the transition amplitude between the initial state and the final state to any desired order,

$$
S_{\mathbf{k} \alpha}=\sum_{n=0}^{\infty} S_{\mathbf{k} \alpha}^{(n)}
$$

with

$$
\begin{aligned}
S_{\mathbf{k} \alpha}^{(0)} & =\mathrm{i}\left\langle\Psi_{X, \mathbf{k}}\left(t_{f}\right)\left|\hat{\mathrm{G}}_{\lambda}^{0}\left(t_{f}, t_{i}\right)\right| \psi_{\alpha}\left(t_{i}\right)\right\rangle, \\
S_{\mathbf{k} \alpha}^{(1)} & =\mathrm{i} \int \mathrm{d} t_{1}\left\langle\Psi_{X, \mathbf{k}}\left(t_{f}\right)\left|\hat{\mathrm{G}}_{X}^{\mathrm{f}}\left(t_{f}, t_{1}\right) V_{X, \lambda}^{0}\left(t_{1}\right) \hat{\mathrm{G}}_{\lambda}^{0}\left(t_{1}, t_{i}\right)\right| \psi_{\alpha}\left(t_{i}\right)\right\rangle .
\end{aligned}
$$

Depending on whether (68) or (69) is substituted in (70) one obtains either

$$
S_{\mathbf{k} \alpha}^{(2)}=\mathrm{i} \iint \mathrm{d} t_{1} \mathrm{~d} t_{2}\left\langle\Psi_{X, \mathbf{k}}\left(t_{f}\right)\left|\hat{\mathrm{G}}_{X}^{\mathrm{f}}\left(t_{f}, t_{2}\right) U \hat{\mathrm{G}}_{\lambda}^{0}\left(t_{2}, t_{1}\right) V_{X, \lambda}^{0}\left(t_{1}\right) \hat{\mathrm{G}}_{\lambda}^{0}\left(t_{1}, t_{i}\right)\right| \psi_{\alpha}\left(t_{i}\right)\right\rangle
$$

or

$$
S_{\mathbf{k} \alpha}^{(2)}=\mathrm{i} \iint \mathrm{d} t_{1} \mathrm{~d} t_{2}\left\langle\Psi_{X, \mathbf{k}}\left(t_{f}\right)\left|\hat{\mathrm{G}}_{X}^{\mathrm{f}}\left(t_{f}, t_{2}\right) U \hat{\mathrm{G}}_{X}^{\mathrm{f}}\left(t_{2}, t_{1}\right) V_{X, \lambda}^{0}\left(t_{1}\right) \hat{\mathrm{G}}_{\lambda}^{0}\left(t_{1}, t_{i}\right)\right| \psi_{\alpha}\left(t_{i}\right)\right\rangle
$$

and so on, where the integration is performed in the range $t_{i}$ to $t_{f}$.

From Eqs. (60) and (61) follows

$$
S_{\mathbf{k} \alpha}^{(0)}=\left\langle\Psi_{X, \mathbf{k}}\left(t_{f}\right) \mid \Psi_{\lambda, \alpha}\left(t_{f}\right)\right\rangle=\mathrm{e}^{\mathrm{i} \Omega_{v \mathbf{k} \alpha}\left(t_{f}\right)} \tilde{\psi}_{\alpha}(\mathbf{k})
$$

where $\tilde{\psi}_{\alpha}(\mathbf{k})=\left\langle\mathbf{k} \mid \psi_{\alpha}\right\rangle$ is the Fourier transform of $\psi_{\alpha}$.

From Eqs. (60) and (49) follows on the other hand

$$
S_{\mathbf{k} \alpha}^{(1)}=\mathrm{i} \int_{t_{i}}^{t_{f}} \mathrm{~d} t\left\langle\Psi_{X, \mathbf{k}}(t)\left|V_{X, \lambda}^{0}(t)\right| \Psi_{\lambda, \alpha}(t)\right\rangle
$$


which — using the identity (64) - can be reduced to

$$
S_{\mathbf{k} \alpha}^{(1)}=\mathrm{i} \int_{t_{i}}^{t_{f}} \mathrm{~d} t\left\langle\mathbf{k}\left|\mathrm{e}^{\mathrm{i} \Omega_{v \mathbf{k} \alpha}(t)} \bar{V}_{v}^{0}(t)\right| \psi_{\alpha}\right\rangle .
$$

In an analogous way, Eq. (74) can be transformed using (54) as

$$
\begin{aligned}
S_{\mathbf{k} \alpha}^{(2)} & =\mathrm{i} \int_{t_{i}}^{t_{f}} \mathrm{~d} t_{2} \int_{t_{i}}^{t_{f}} \mathrm{~d} t_{1}\left\langle\Psi_{X, \mathbf{k}}\left(t_{2}\right)\left|U \hat{\mathrm{G}}_{\lambda}^{0}\left(t_{2}, t_{1}\right) V_{X, \lambda}^{0}\left(t_{1}\right)\right| \psi_{\lambda, \alpha}\left(t_{1}\right)\right\rangle \\
& =\int_{t_{i}}^{t_{f}} \mathrm{~d} t_{2} \sum_{\alpha^{\prime}}\left\langle\Psi_{X, \mathbf{k}}\left(t_{2}\right)|U| \Psi_{\lambda, \alpha^{\prime}}\left(t_{2}\right)\right\rangle \int_{t_{i}}^{t_{2}} \mathrm{~d} t_{1}\left\langle\Psi_{\lambda, \alpha^{\prime}}\left(t_{1}\right)\left|V_{X, \lambda}^{0}\left(t_{1}\right)\right| \Psi_{\lambda, \alpha}\left(t_{1}\right)\right\rangle \\
& =\int_{t_{i}}^{t_{f}} \mathrm{~d} t_{2} \sum_{\alpha^{\prime}}\left\langle\mathbf{k}\left|\mathrm{e}^{\mathrm{i} \Omega_{v \mathbf{k} \alpha^{\prime}}\left(t_{2}\right)} U\right| \psi_{\alpha^{\prime}}\right\rangle \int_{t_{i}}^{t_{2}} \mathrm{~d} t_{1} \mathrm{e}^{\mathrm{i}\left(E_{\alpha^{\prime}}-E_{\alpha}\right) t_{1}}\left\langle\psi_{\alpha^{\prime}}\left|\bar{V}_{v}^{0}\left(t_{1}\right)\right| \psi_{\alpha}\right\rangle
\end{aligned}
$$

or Eq. (75) can be transformed using (48) as

$$
\begin{aligned}
S_{\mathbf{k} \alpha}^{(2)} & =\mathrm{i} \int_{t_{i}}^{t_{f}} \mathrm{~d} t_{2} \int_{t_{i}}^{t_{f}} \mathrm{~d} t_{1}\left\langle\Psi_{X, \mathbf{k}}\left(t_{2}\right)\left|U \hat{\mathrm{G}}_{X}^{\mathrm{f}}\left(t_{2}, t_{1}\right) V_{X, \lambda}^{0}\left(t_{1}\right)\right| \psi_{\lambda, \alpha}\left(t_{1}\right)\right\rangle \\
& =\int_{t_{i}}^{t_{f}} \mathrm{~d} t_{2} \sum_{\mathbf{k}^{\prime}}\left\langle\Psi_{X, \mathbf{k}}\left(t_{2}\right)|U| \Psi_{X, \mathbf{k}^{\prime}}\left(t_{2}\right)\right\rangle \int_{t_{i}}^{t_{2}} \mathrm{~d} t_{1}\left\langle\Psi_{X, \mathbf{k}^{\prime}}\left(t_{1}\right)\left|V_{X, \lambda}^{0}\left(t_{1}\right)\right| \Psi_{\lambda, \alpha}\left(t_{1}\right)\right\rangle \\
& =\int_{t_{i}}^{t_{f}} \mathrm{~d} t_{2} \sum_{\mathbf{k}^{\prime}} \mathrm{e}^{\mathrm{i}\left(E_{k}-E_{k^{\prime}}\right) t_{2}+\mathrm{i}\left(\mathbf{k}-\mathbf{k}^{\prime}\right) \cdot \boldsymbol{\alpha}\left(t_{2}\right)}\left\langle\mathbf{k}|U| \mathbf{k}^{\prime}\right\rangle \int_{t_{i}}^{t_{2}} \mathrm{~d} t_{1}\left\langle\mathbf{k}^{\prime}\left|\mathrm{e}^{\mathrm{i} \Omega_{v \mathbf{k}^{\prime} \alpha}\left(t_{1}\right)} \bar{V}_{v}^{0}\left(t_{1}\right)\right| \psi_{\alpha}\right\rangle .
\end{aligned}
$$

Continuing in an analogous manner, it can be shown that $S_{\mathbf{k} \alpha}^{(n)}$ for any order $n$ depends only on $v$. Therefore, $S_{\mathbf{k} \alpha}$ itself depends only on $v$. In the next subsection some particular cases will be considered explicitly.

\section{Particular cases}

As a first example, consider the case studied in [14]. It is obtained using $v=\{0,0\}$ where one has

$$
\begin{aligned}
\bar{V}_{v}^{0}(t) & =\mathbf{F}(t) \cdot \mathbf{r} \\
\Omega_{v \mathbf{k} \alpha}(t) & =\left(E_{k}-E_{\alpha}\right) t+\mathbf{k} \cdot \boldsymbol{\alpha}(t)-\mathbf{A}(t) \cdot \mathbf{r}+\beta(t) .
\end{aligned}
$$

This formulation is achieved using the following partitionings for different gauges:

$$
\lambda=\{-1,0\} \text { in R-gauge, } \quad \lambda=\{-1,1\} \text { in V-gauge, } \quad \lambda=\{0,0\} \text { in L-gauge. }
$$

Since in L gauge the relation $\hat{\mathrm{H}}_{\lambda}^{0}=\hat{\mathrm{H}}^{0}$ holds, the gauge-invariant formulation with $v=\{0,0\}$ reproduces the traditional SFA in the L gauge.

However, in an analogous way, the traditional V-gauge SFA is obtained with $v=\{1,-1\}$ (cf. [19]), where

$$
\begin{aligned}
\bar{V}_{v}^{0}(t) & =\mathbf{A}(t) \cdot \mathbf{p}_{\mathrm{c}} \\
\Omega_{v \mathbf{k} \alpha}(t) & =\left(E_{k}-E_{\alpha}\right) t+\mathbf{k} \cdot \boldsymbol{\alpha}(t) .
\end{aligned}
$$

It can be achieved using the following partitionings for different gauges:

$$
\lambda=\{0,-1\} \text { in R-gauge, } \quad \lambda=\{0,0\} \text { in V-gauge, } \quad \lambda=\{1,-1\} \text { in L-gauge. }
$$

In a similar way, the traditional R-gauge SFA is obtained with $v=\{1,0\}$, where

$$
\begin{aligned}
\bar{V}_{v}^{0}(t) & =\mathbf{A}(t) \cdot \mathbf{p}_{\mathrm{c}}+\mathbf{A}^{2}(t) / 2 \\
\Omega_{v \mathbf{k} \alpha}(t) & =\left(E_{k}-E_{\alpha}\right) t+\mathbf{k} \cdot \boldsymbol{\alpha}(t)+\beta(t) .
\end{aligned}
$$

It can be achieved using the following partitionings for different gauges:

$$
\lambda=\{0,0\} \text { in R-gauge, } \quad \lambda=\{0,1\} \text { in V-gauge, } \quad \lambda=\{1,0\} \text { in L-gauge. }
$$

Clearly, every $S$-matrix expansion (SFA formulation) in one of the "conventional" (length, velocity, or radiation) gauges can be obtained by adopting any of the possible gauges, if the partitioning of the Hamiltonian is chosen accordingly. 


\section{CONCLUSION}

In this work it is shown how the (infinite-order) $S$-matrix expansion describing atomic or molecular systems in intense laser fields depends on the choice of both the gauge and the partitioning of the Hamiltonian. For this purpose a generalized gauge as well as a generalized partioning scheme is introduced. They are defined by 4 independent parameters $\left(x_{1}\right.$ and $x_{2}$ for the gauge as well as $\lambda_{1}$ and $\lambda_{2}$ for the partitioning). However, the $S$-matrix expansion is then shown to depend only on 2 parameters, $v_{1}=1+\lambda_{1}-x_{1}$ and $v_{2}=\lambda_{2}-x_{2}$. Clearly, every possible combination of the parameters defining the gauge and the partitioning that conserves the values $\lambda_{1}-x_{1}$ and $\lambda_{2}-x_{2}$ leads to an identical $S$-matrix expansion (up to an overall phase factor that cancels when calculating physical observables).

The present analysis thus shows that one has to be very careful not to consider solely the gauge, since a suitable choice of the partitioning may lead to an $S$-matrix expansion that is identical to the one obtained in some other gauge, as was also demonstrated in [14] where the velocity-gauge expansion appeared to agree with what is usually known as length-gauge $S$-matrix expansion. In fact, it is much more appropriate to discuss different expansions than different gauges. This avoids for example the rather contradictory terminology of a "gauge-invariant (first-order) KFR approximation in the velocity gauge" used in [14].

Furthermore, the present result demonstrates that there remains an in principle infinite set of $S$-matrix expansions (characterized by different values of $v_{1}, v_{2}$ ) that are only shown to provide the same transition probabilities in the limit of an infinite series expansion, if the latter converges. Truncated series like, e.g., the 0th, 1st, or 2nd order expansions will, however, in general disagree. Thus the question of a "proper" choice of the expansion in the case of truncation remains and can only be clarified by a comparison to either experiment or gauge-independent theory (like full solutions of the time-dependent Schrödinger equation). It should be emphasized, however, that there is no a priori reason to believe that one expansion is necessarily advantageous to the others.

\section{Acknowledgments}

The authors would like to thank H. R. Reiss for in-depth discussions. This work was supported by the Deutsche Forschungsgemeinschaft. AS is grateful to the Stifterverband für die Deutsche Wissenschaft (Programme Forschungsdozenturen) and the Fonds der Chemischen Industrie for financial support.

[1] L. V. Keldysh, Sov. Phys. JETP 20, 1307 (1965).

[2] F. H. M. Faisal, J. Phys. B: At. Mol. Phys. 6, L89 (1973).

[3] H. R. Reiss, Phys. Rev. A 22, 1786 (1980).

[4] J. Bauer, Phys. Rev. A 73, 023421 (2006).

[5] Y. V. Vanne and A. Saenz, Phys. Rev. A 75, 063403 (2007).

[6] D. Bauer, D. B. Milošević, and W. Becker, Phys. Rev. A 72, 023415 (2005).

[7] J. Muth-Böhm, A. Becker, and F. H. M. Faisal, Phys. Rev. Lett. 85, 2280 (2000).

[8] T. K. Kjeldsen and L. B. Madsen, J. Phys. B: At. Mol. Phys. 37, 2033 (2004).

[9] D. B. Milošević, Phys. Rev. A 74, 063404 (2006).

[10] M. Awasthi, Y. V. Vanne, A. Saenz, A. Castro, and P. Decleva, Phys. Rev. A 77, 063403 (2008).

[11] T. K. Kjeldsen and L. B. Madsen, Phys. Rev. A 71, 023411 (2005).

[12] B. Bergues, Z. Ansari, D. Hanstorp, and I. Y. Kiyan, Phys. Rev. A 75, 063415 (2007).

[13] H. R. Reiss, Phys. Rev. A 76, 033404 (2007).

[14] F. H. M. Faisal, J. Phys. B: At. Mol. Phys. 40, F145 (2007).

[15] A. Becker and F. H. M. Faisal, J. Phys. B: At. Mol. Phys. 38, R1 (2005).

[16] D. B. Milošević, G. G. Paulus, D. Bauer, and W. Becker, J. Phys. B: At. Mol. Phys. 39, R203 (2006).

[17] H. R. Reiss, Phys. Rev. A 77, 067401 (2008).

[18] B. Bergues, Z. Ansari, D. Hanstorp, and I. Y. Kiyan, Phys. Rev. A 77, 067402 (2008).

[19] F. H. M. Faisal, Phys. Rev. A 75, 063412 (2007). 